\title{
In Memoriam Prof. Dr. med. Gerhard Neumann
}

\author{
In Memoriam Prof. Dr. med. Gerhard Neumann
}

Autoren

Institute
M. Priwitzer ${ }^{1}$, R. Loddenkemper ${ }^{2}$

1 Gesundheitsamt Stuttgart

2 Generalsekretär des DZK

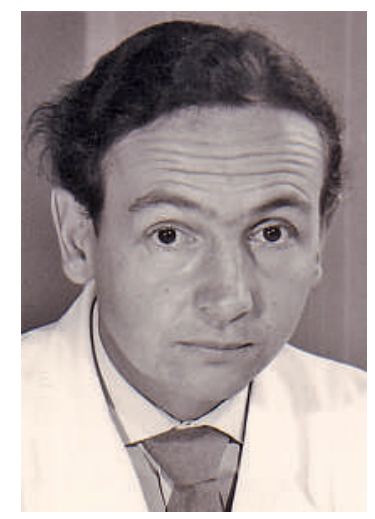

Prof. Dr. med. Gerhard Neumann

Bibliografie

DOI 10.1055/s-0028-1119690

Pneumologie 2009; 63: 182-183

(c) Georg Thieme Verlag KG

Stuttgart · New York

ISSN 0934-8387

Korrespondenzadresse

Dr. Martin Priwitzer

Landeshauptstadt Stuttgart Gesundheitsamt

Bismarckstr. 3

70176 Stuttgart

Martin.Priwitzer@stuttgart.de
Am 12. Dezember 2008, wenige Wochen nach seinem 89. Geburtstag, starb Prof. Dr. Gerhard Neumann, der frühere Leiter des Gesundheitsamtes der Landeshauptstadt Stuttgart. Mit ihm verliert der öffentliche Gesundheitsdienst einen Arzt und Wissenschaftler, der der Entwicklung der Tuberkulosefürsorge und der epidemiologischen Forschung im Nachkriegsdeutschland entscheidende Impulse gegeben hat.

Gerhard Neumann, 1919 in Reichenbach in der Oberlausitz geboren, ist den mühevollen Weg zahlreicher Kollegen seiner Generation gegangen: Nach dem Abitur im Frühjahr 1938 wurde er erst zum Reichsarbeitsdienst, dann zur Wehrmacht einberufen, als Kradmelder, wie er einmal erzählte: Mit dem Motorrad als Bote unterwegs und dabei ganz auf sich alleine gestellt, habe er es schnell gelernt, sich stets einen Rückzugsweg offen zu halten, um zwischen den Fronten ungeschoren hindurch zu kommen - eine prägende Erfahrung für einen jungen Menschen. Von 1940 bis 1947 studierte er in Breslau und Göttingen Medizin und war nach der Notapprobation und Promotion im April 1945 zunächst als Unterarzt in der Kriegsgefangenschaft tätig. Bis zur Vollapprobation 1947 folgten Tätigkeiten als Volontärarzt im Städtischen Krankenhaus in Verden an der Aller und in einer Allgemeinarztpraxis. Die Weiterbildung zum Lungenfacharzt absolvierte er in den Heilstätten Kalandshof in Rotenburg (Wümme) und Erbprinzentanne in ClausthalZellerfeld. 1954 wechselte Gerhard Neumann als Tuberkulosefürsorgearzt in das Gesundheitsamt Stuttgart. Während es heute immer schwieriger wird, Lungenfachärzte für den öffentlichen Gesundheitsdienst zu gewinnen, war der Weg in ein Gesundheitsamt für einen jungen Pneumologen damals nicht ungewöhnlich. Schon bald erkannten seine Vorgesetzten, dass in dem jungen Arzt ein Potenzial schlummerte, das über die tägliche Routinearbeit in der Sprechstunde weit hinausging. So war es kein Wunder, dass er die
Hierarchieleiter fast zwangsläufig und ohne eigenen Ehrgeiz empor kletterte, bis er 1975 vom Gemeinderat zum Amtsleiter gewählt wurde, und gleichzeitig seinem wissenschaftlichen Interesse freien Lauf ließ.

Gerhard Neumann, der kleine, drahtige Mann, der die Tuberkulose am eigenen Leib erfahren musste, hatte ein durchaus inniges Verhältnis zu seinen Patienten; da war er ganz Arzt, nicht Wissenschaftler, und bis ins hohe Alter konnte man spüren, dass sein Herz den Tuberkulosekranken gehörte. Tuberkulosefürsorge bedeutete für ihn in erster Linie die ärztliche Aufgabe, Krankheiten zu erkennen und die Kranken zu betreuen. Gleichzeitig jedoch gewann er schon in jungen Jahren schnell ein Gespür für epidemiologische und seuchenhygienische Fragestellungen. Er verstand es, die Flut an Daten, die in der täglichen Arbeit eines großen Gesundheitsamtes anfällt, zu kanalisieren, sinnvoll auszuwerten und aus den Ergebnissen die richtigen Schlussfolgerungen zu ziehen. Dadurch prägte er bis in die 80erJahre hinein den öffentlichen Gesundheitsdienst in Deutschland.

Allein zum Thema Tuberkulose veröffentlichte Gerhard Neumann mehr als 100 in PubMed gelistete wissenschaftliche Veröffentlichungen. Dem Deutschen Zentralkomitee zur Bekämpfung der Tuberkulose (DZK), aber auch der Deutschen Gesellschaft für Pneumologie (DGP) war er über Jahrzehnte ein äußerst wichtiger Berater und federführend an der Ausarbeitung mehrerer Expertisen zur Tuberkulose beteiligt. Für seine Verdienste um die Pneumologie, und besonders um die Tuberkulose, wurde er zum Ehrenmitglied sowohl des DZK als auch der DGP ernannt.

Von seinem Vorgänger in der Stuttgarter Tuberkulosefürsorge übernahm Gerhard Neumann eine gut aufgestellte Abteilung, die eine auch internationalem Standard entsprechende Arbeit ermöglichte. Er ergriff die Gelegenheit, die dortigen Erfahrungen systematisch auszuwerten und 
zu veröffentlichen. Seine Mitarbeiter schätzten sein Bemühen, nicht nur selbst wissenschaftlich zu arbeiten, sondern auch ihnen die Teilnahme an Kongressen, Tagungen, nationalen und internationalen Studien zu ermöglichen.

Die durch die Forschungsarbeit entstandenen internationalen Kontakte, vor allem in die angelsächsischen Länder und in die Niederlande, erweiterten seinen Blick auf andere Gesundheitssysteme. In der Folge verschob sich sein wissenschaftlicher Interessensschwerpunkt zu anderen epidemiologischen Fragestellungen, insbesondere im Zusammenhang mit der Krebsbekämpfung. Er war maßgebend an der Einführung der Krebsfrüherkennung in Württemberg und später an der Einrichtung eines Krebsregisters in Baden-Württemberg beteiligt. Lange Jahre war er Geschäftsführer des Landesverbandes Baden-Württemberg zur Erforschung und Bekämpfung des Krebses (heute Krebsverband Baden-Württemberg). Auch heute noch zitiert wird seine grundlegende Arbeit über „Mortalität und Sozialschicht" von 1981, in der er den Zusammenhang zwischen dem sozialen Status - repräsentiert durch den Beruf - und der Sterblichkeit darstellte.

1969 habilitierte sich Gerhard Neumann an der Universität Heidelberg für das Fach Sozialmedizin und wurde 1973 zum außerordentlichen Professor ernannt. 1973 und 1975 lehnte er einen Ruf auf die Professur für Sozialmedizin an der Universität Gießen ab. Ein Hauptgrund dafür war, dass er, wie er in seiner Abschiedsrede als Amtsleiter augenzwinkernd bemerkte, schon seit längerem die Mainlinie lieber von Norden nach Süden überquerte als umgekehrt; Stuttgart war zu seiner zweiten Heimat geworden.
Bis wenige Monate vor seinem Tod kam Gerhard Neumann regelmäßig in seine „alte“ Abteilung im Gesundheitsamt, um einige Zeitschriften abzugeben, wenn sie sich zu Hause im Wohnzimmer wieder türmten. Dabei fragte er stets nach der aktuellen Fallarbeit und hatte Freude daran, hin und wieder eine diffizile Röntgenserie gemeinsam unter die Lupe zu nehmen oder sich über die neuesten Entwicklungen auszutauschen, sei es das Fingerprinting, seien es die Interferon-gamma-Tests. Als Gutachter für die Sozialgerichte vergrub er sich auch noch in seinen Achtzigern manchmal stundenlang in die Abteilungsbibliothek, wenn er für ein schwieriges Sozialgerichtsgutachten aktuelle Literatur benötigte. Bis ins hohe Alter blieb Gerhard Neumann geistig beweglich. Er, der wie viele Tuberkulose-Experten seiner Generation in den 70er- und 80er-Jahren noch das Verschwinden der Tuberkulose in Deutschland und Europa binnen weniger Jahrzehnte prophezeit hatte, war sich nicht zu schade, seine Fehleinschätzung zu revidieren, und erkannte die Notwendigkeit, auf eine veränderte epidemiologische Situation mit neuen Ideen und Maßnahmen zu reagieren. Für die Zeitschrift „Pneumologie" schrieb er noch im Ruhestand kritische Reviews zu internationalen Veröffentlichungen zum Thema Tuberkulose, die sein großes Wissen um diese Krankheit widerspiegelten.

Gerhard Neumann veröffentlichte über 300 wissenschaftliche Arbeiten. Er war in verschiedenen Funktionen für das DZK, für die WHO, für die IUATLD, für die Süddeutsche Gesellschaft für Tuberkulose und Lungenkrankheiten, für das Institut für medizinische und pharmazeutische Prüfungsfragen, für die Bundesund Landesregierung und für andere Organisationen tätig. 1961 erhielt er den Franz-Redeker-Preis des DZK, 1979 das Verdienstkreuz 1. Klasse des Verdienstordens der Bundesrepublik Deutschland. 